\title{
Determination of the magnetic penetration depth in a superconducting $\mathrm{Pb}$ film
}

\author{
J. Brisbois, ${ }^{1, a)}$ B. Raes, ${ }^{2, b)}$ J. Van de Vondel, ${ }^{2}$ V. V. Moshchalkov, ${ }^{2}$ and A. V. Silhanek ${ }^{1}$ \\ ${ }^{1}$ Département de Physique, Université de Liège, B-4000 Sart Tilman, Liège, Belgium \\ ${ }^{2}$ INPAC-Institute for Nanoscale Physics and Chemistry, Nanoscale Superconductivity and Magnetism Group, \\ K.U. Leuven, Celestijnenlaan 200D, B-3001 Leuven, Belgium
}

(Received 19 December 2013; accepted 28 February 2014; published online 12 March 2014)

\begin{abstract}
By means of scanning Hall probe microscopy technique, we accurately map the magnetic field pattern produced by Meissner screening currents in a thin superconducting $\mathrm{Pb}$ stripe. The obtained field profile allows us to quantitatively estimate the Pearl length $\Lambda$ without the need of pre-calibrating the Hall sensor. This fact contrasts with the information acquired through the spatial field dependence of an individual flux quantum where the scanning height and the magnetic penetration depth combine in a single inseparable parameter. The derived London penetration depth $\lambda_{\mathrm{L}}$ coincides with the values previously reported for bulk $\mathrm{Pb}$ once the kinetic suppression of the order parameter is properly taken into account. (C) 2014 AIP Publishing LLC. [http://dx.doi.org/10.1063/1.4868298]
\end{abstract}

\section{INTRODUCTION}

The thermodynamic hallmark of superconductivity is the ability to expel a magnetic field. This phenomenon is produced by macroscopic screening currents circulating at the border of the superconducting material, which in turn generate a magnetic field counteracting the applied one in the interior of the superconductor. In finite size samples, the shielding (or Meissner) currents lead to a reinforcement of the field at the border of the sample usually accounted for through a demagnetization tensor. Since $\nabla \times \vec{B}=\mu_{0} \vec{J}$, a perfect screening with a discontinuous jump in the magnetic field at the sample's borders would need unrealistic diverging currents. In reality, finite screening currents run in a thin layer close to the sample's surface and the field penetrates into the superconductor over a material dependent distance $\lambda$ called the magnetic penetration depth. Its determination is of fundamental importance as it is closely related to parameters relevant for applications, like the lower critical field $H_{\mathrm{c} 1}$ at which penetration of flux quanta becomes energetically favourable, or the depairing current density $J_{\mathrm{dp}}$ corresponding to the maximum current that a superconductor can sustain before restoring the normal metallic behavior.

An absolute determination of $\lambda$ is regarded as a very challenging experimental work. In general, the available techniques can be grouped in two broad categories, namely bulk integrated-response techniques and local probe techniques. Bulk techniques ${ }^{1-6}$ average the response of the whole sample and therefore are not sensitive to the local details such as possible material inhomogeneities. Local techniques $^{7-12}$ not only allow one to gain insights at the microscopic level but can be also considered as a more direct way to assess $\lambda$. Within the local probe category, the most

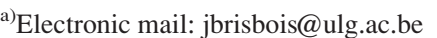

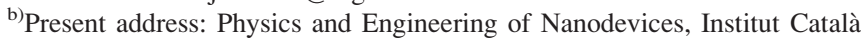
de Nanotecnologia (ICN), Campus de la UAB, Edifici ICN2, 08193 Bellaterra, Spain.
}

popular approach for estimating $\lambda$ consists of mapping the field profile of an isolated flux quantum and track its temperature evolution. Unfortunately, irrespective of whether the flux quantum is an Abrikosov vortex present in a thick sample, or a Pearl vortex ${ }^{13}$ characteristic of thin films, the vertical component of the magnetic field picked up by the sensor depends on a single variable, $z_{0}+\lambda$, forming an indissociable additive combination of the vertical separation between the sensor and the surface of the superconductor, $z_{0}$, and $\lambda$. Therefore, the precision of the extracted $\lambda$ is directly linked to the quality of the calibration of the z-positioners and relies on perfect knowledge of the geometrical configuration and the sensor mounting.

In this study, we propose an alternative method to determine the penetration depth $\lambda$ which has not been explored so far and does not require pre-calibration procedures of the magnetic sensor. Its precision depends on the good knowledge of the sample geometry. The approach consists of mapping the magnetic field profile at the border of the sample produced by Meissner screening currents. In contrast to the centrosymmetric field landscape of an isolated vortex, now the contribution of the vertical separation $z_{0}$ and the penetration depth contribute separately to the vertical component of the detected magnetic field, thus facilitating the estimation of $\lambda$. Even though the method is illustrated with a particular magnetic probe (i.e., a Hall sensor), it is applicable to any other scanning magnetic probe techniques such as magnetic force microscopy (MFM) or SQUID microscopy. In addition, this method can also work for current-driven transport bridges at zero external field.

\section{EXPERIMENTAL DETAILS}

We study the response of a $\mathrm{Pb}$ strip of length $L=3 \mathrm{~mm}$, width $2 a=600 \mu \mathrm{m}$, and thickness $t=50 \mathrm{~nm}$ deposited on an insulating $\mathrm{SiO}_{2} / \mathrm{Si}$ substrate. The surface is protected by a $60 \mathrm{~nm}$ thick insulating Ge layer to prevent oxidation. The sample is patterned with a square lattice of square holes of 
side length $b=600 \mathrm{~nm}$ and period $w=3 \mu \mathrm{m}$, as schematically shown in Fig. 1. As we discuss below, the ratio $b / w$ has been chosen in such a way that the holes have negligible effect on the penetration length while allowing us to readily check the onset of flux penetration into the sample, i.e., the limit of the Meissner state. A superconducting critical temperature $T_{\mathrm{c}}=7.2 \mathrm{~K}$ was determined by ac-susceptibility measurements. ${ }^{14}$ A $50 \mathrm{~nm}$ thick gold layer covers the sample to allow using a tunneling conducting tip as a feedback loop to approach the sample surface.

The Hall probe is a cross-shaped 2DEG (GaAs/AlGaAs) scanning alongside the sample surface, fed with a constant dc current $\sim 20 \mu \mathrm{A}$, and with readout voltage proportional to the component of the magnetic field perpendicular to the probe. Scanning Hall probe microscopy (SHPM) exhibits several advantages for our experiment: it is noninvasive, it offers a good compromise between spatial and field resolution ${ }^{15}$ and, in lift mode, it is a relatively fast way to get two-dimensional images of the magnetic field. We use a commercial lowtemperature SHPM (NanoMagnetics Instruments) modified by adding a piezoelectric horizontal slider to allow the adjustment of the sample position. The vertical position is adjusted by a piezoelectric slider (coarse positioning of step $p \sim 0.8 \mu \mathrm{m}$ according to the specifications of the manufacturer) and a piezoelectric tube (fine positioning).

A standard approaching process ${ }^{16}$ ends when a tunnel current of $0.5 \mathrm{nA}$ is obtained, corresponding to a tunnel resistance of $200 \mathrm{M} \Omega$ and a tip-sample distance smaller than $1 \mathrm{~nm}$. Once the tip is in STM-contact with the sample, the ultimate lower limit for the height above the sample surface is determined by the depth of the 2DEG below the surface of the heterostructure, typically $100 \mathrm{~nm}$. However, an additional and far more important limitation comes from the angle between the sample and probe chip $\sim 1^{\circ}-2^{\circ}$ and the fact that the Hall sensor is about $15 \mu \mathrm{m}$ away from the STM tip as schematically shown in Fig. 2. The distance between the active area and the STM tip combined with the tilt angle contributes to the height of the probe. In principle, it is possible to scan the sensor over the sample surface while keeping

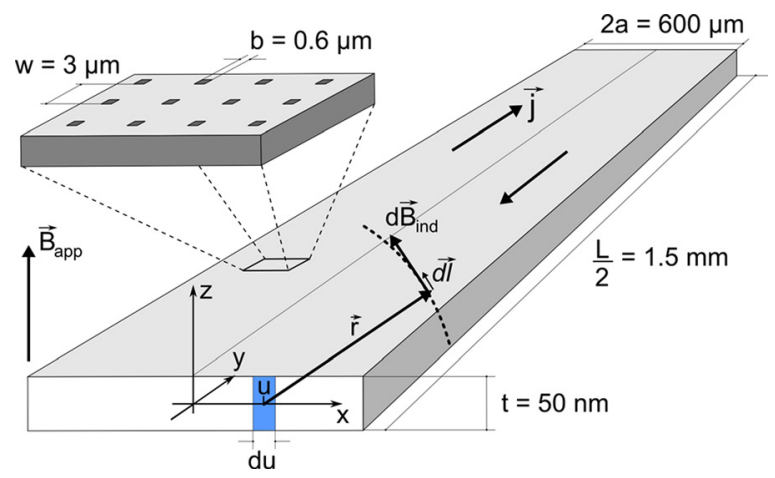

FIG. 1. Sketch of the nanostructured thin superconducting Pb film of thickness $t=50 \mathrm{~nm}$. The film has a length $L$ and a width $2 a$ and it is patterned by a square lattice (period $w$ ) of square holes (side-length $b$ ). In the Meissner state, when a constant magnetic field $B_{\text {app }}$ is applied, screening currents $\vec{j}(u)$ flow in the sample. Each infinitesimal element $d u$ centred on $u$ generates a contribution $d \vec{B}_{\text {ind }}$ to the magnetic field $\vec{B}_{\text {ind }}$. The total field is calculated by Biot-Savart's law and is the sum of $\vec{B}_{\text {app }}$ and the contribution of the screening currents.

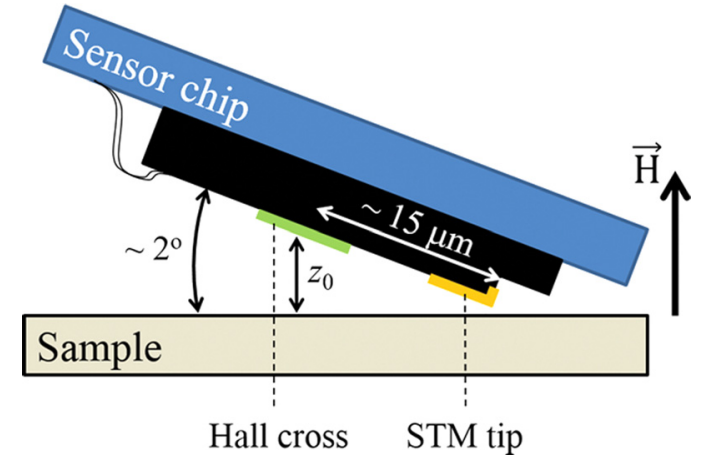

FIG. 2. Schematic illustration of the sample-sensor chip orientation and position. In this configuration, the STM tip is always the point of the Hall sensor closest to the sample, thus limiting the risk of crashing the probe during the approach. The minimal distance $z_{0}$ is limited by the depth of the 2DEG in the heterostructure and by the distance between the STM tip and the Hall cross. Moreover, the sensor is usually lifted by a few hundreds of $\mathrm{nm}$ to allow fast imaging.

the tunneling current constant using a PID protocol on the z-piezo, obtaining simultaneously the topography and the magnetic field distribution. In this configuration, the Hall sensor scans over the sample surface at a minimum distance of $600 \mathrm{~nm}$. However, in practice, after STM-contact at the highest corner of the scan area, we lift the scan head a few hundreds of $\mathrm{nm}$ and the scan is performed at this fixed distance $z_{0} \sim 1 \mu \mathrm{m}$. The lift height is limited by the surface roughness within the scan area and can be continuously adjusted during scanning in both lateral and transversal directions for compensating the tilt of the sample. This liftmode allows for faster imaging, with less risk of crashing the probe. In this work, keeping in mind the aforementioned restrictions, we introduce a protocol to precisely determine the height of the Hall sensor $z_{0}$. Moreover, this characterization allows us to obtain a good estimate for $\lambda_{\mathrm{L}}$.

\section{FIELD PROFILE PRODUCED BY MEISSNER CURRENTS}

\section{A. Theoretical model}

Let us start analyzing the magnetic response of a superconducting strip in the Meissner state as shown in Fig. 1. The strip occupies the space $|x| \leq a,|y| \leq L / 2 \gg a$, and $|z| \leq t / 2 \ll a$. After cooling down the sample below $T_{\mathrm{c}}$, a constant magnetic field $H_{\text {app }}=B_{\text {app }} / \mu_{0}$ is applied perpendicularly to the surface (zero-field cooling process). Consequently, screening currents flow around the strip in an attempt to shield $H_{\text {app }}$ inside the superconductor. Assuming that $t<\lambda$, the current density is nearly independent of $z$ across the film thickness, and therefore it is convenient to consider only the surface current density $\vec{J}_{\mathrm{S}}(x, y)$ defined by averaging the volume current density $\vec{j}(x, y, z)$ over the thickness of the strip,

$$
\vec{J}_{\mathrm{S}}(x, y)=\int_{-t / 2}^{t / 2} \vec{j}(x, y, z) d z .
$$

The total magnetic field $\vec{B}_{\text {tot }}(x, y, z)$ at the point of coordinates $(x, y, z)$ is the sum of two contributions: the applied 
field $\vec{B}_{\text {app }}$ and the field generated by the screening currents $\vec{B}_{\text {ind }}(x, y, z)$. As $L \gg a, t, \vec{B}_{\text {tot }}$ and $\vec{J}_{\mathrm{S}}$ do not depend on $y$. In what follows, the only component of $\vec{B}_{\text {tot }}(x, z)$ considered relevant for measurements with magnetic probes is the $z$-component $B_{z}(x, z)$. We can work out an expression for $\vec{B}_{\text {ind }}(x, z)$ based on the notations of Fig. 1.

The field $d \vec{B}_{\text {ind }}$ produced by an infinitesimal portion of material $d u$ crossed by a current $I(u)$ can be determined by Ampère's law, and by subsequently summing up the contribution of each infinitesimal portion $d u$, we obtain

$$
B_{\text {ind }, z}(x, z)=\frac{\mu_{0}}{2 \pi} \int_{-a}^{a} \frac{(x-u) J_{\mathrm{L}}(u) d u}{(x-u)^{2}+z^{2}},
$$

where $J_{\mathrm{L}}$ is the linear current density defined as $J_{\mathrm{L}}(x)$ $=J_{\mathrm{S}}(x, y) d y$. The $z$-component of $\vec{B}_{\text {tot }}$ at $(x, y, z)$ is thus given by

$$
B_{z}(x, z)=\frac{\mu_{0}}{2 \pi} \int_{-a}^{a} \frac{(x-u) J_{\mathrm{L}}(u) d u}{(x-u)^{2}+z^{2}}+B_{\mathrm{app}}
$$

An expression for $J_{\mathrm{L}}(u)$ obtained from the London-Maxwell equations for a thin $(t \ll a)$ rectangular strip is given by Plourde et al. ${ }^{17}$ for a constant magnetic field perpendicular to the surface. When $a / \Lambda \gg 1$ where $\Lambda=\lambda^{2} / t$ is the Pearl length, $J_{\mathrm{L}}(u)$ is expressed as

$$
J_{\mathrm{L}}(u)=\frac{2 u B_{\text {app }}}{\mu_{0} \sqrt{\left(a^{2}-u^{2}\right)+\frac{4 a}{\pi} \Lambda}} .
$$

By inserting this relationship in Eq. (3), we can deduce the relative change of magnetic field as the Hall probe scans the width of the strip at a height $z$ :

$$
\frac{B_{z}(x, z)}{B_{\text {app }}}=1+\frac{1}{\pi} \int_{-a}^{a} \frac{(x-u) u d u}{\left((x-u)^{2}+z^{2}\right) \sqrt{\left(a^{2}-u^{2}\right)+\frac{4 a}{\pi} \Lambda}} .
$$

In Sec. III B, we will use this equation to model the $B_{z}(x, z)$ profiles obtained experimentally at different distances $z$ from the surface of the superconductor.

\section{B. Experimental results}

In order to obtain the magnetic field profile in the Meissner state, a series of SHPM images were recorded at the border of the $\mathrm{Pb}$ strip at $T=4.2 \mathrm{~K}$ and $H_{\text {app }}=B_{\text {app }} / \mu_{0}$ in zero field cooling. An example of the resulting field landscape is shown in Fig. 3(a). The border of the strip can easily be localized by the bright strip corresponding to the magnetic field generated by the Meissner screening currents. For each image, we averaged ten cross-sections taken perpendicularly to the border in order to reduce the noise-to-signal ratio of the field profiles. We then divide the result by $B_{\text {app }}$ and fit the results following Eq. (5). This equation indicates that the larger the applied field, the bigger will be the signal detected and therefore higher signal-to-noise ratio can be achieved. This argument holds for fields below a certain threshold $H_{\mathrm{p}}$ at which flux quanta penetrates into the sample. ${ }^{14}$ Since the
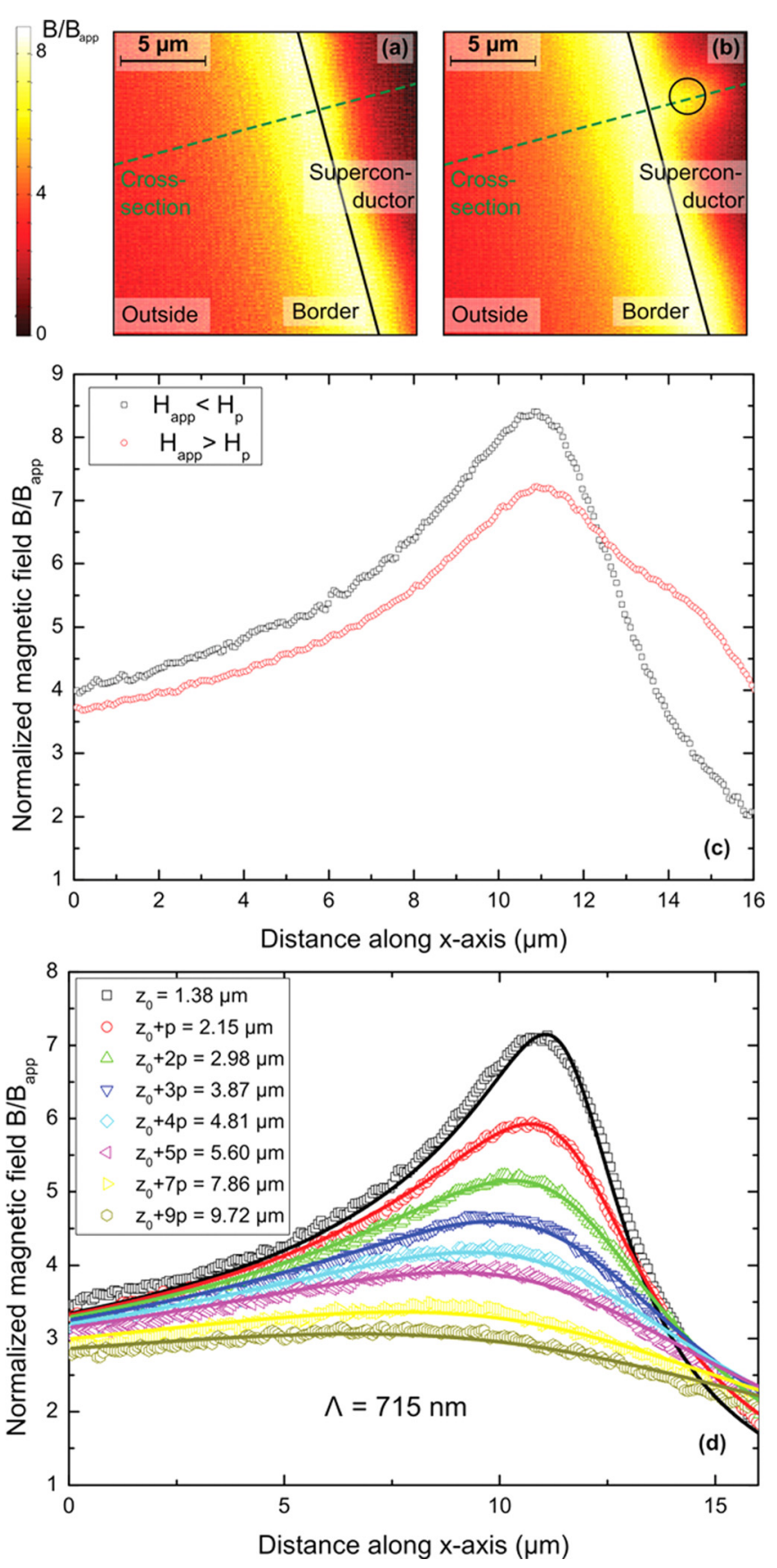

FIG. 3. (a) and (b) show the SHPM images of the superconducting strip border in the Meissner state in a constant magnetic field $H_{\text {app }}$, respectively, lower and slightly higher than $H_{\mathrm{p}}$, the field corresponding to the first vortex entry in the sample. The bright area indicates the higher magnetic field and is due to the presence of demagnetizing effects at the border of the sample (black line). In panel (b), a vortex trapped by an antidot close to the sample border has been highlighted with a circle. The cross-sections shown in (c) were taken along the green lines. (d) Influence of the distance $z$ between the Hall probe and the sample surface on the normalized magnetic field profile at the border of the strip. $z_{0}$ is the minimum scanning height and $p$ is the stepsize of the vertical piezo slider. The curves computed numerically (continuous lines) from the relation (5) are superposed to the data. In addition to the scanning heights, the model yields a value for the transversal penetration depth $\Lambda=715 \pm 300 \mathrm{~nm}$.

holes act as pinning centers preventing vortices to proceed towards the middle of the sample, the pinned vortices contribute to the field measured at the border of the sample and serve as an indication that the condition $H_{\text {app }}>H_{\mathrm{p}}$ has been 
achieved. This is illustrated in Fig. 3(b) where a vortex is indicated by a black circle. In Fig. 3(c), we show the field profile for $H_{\text {app }} \lesssim H_{\mathrm{p}}$ and $H_{\text {app }} \gtrsim H_{\mathrm{p}}$. Notice that the penetration of a flux quantum relaxes the magnetic field at the border of the sample.

Once the value of $H_{\mathrm{p}}$ has been identified, Eq. (5) can be used to model the field profiles at different heights $z$ obtained for $H_{\text {app }} \leq H_{\mathrm{p}}$. The experimental data are plotted in Fig. 3(d). As $z$ increases, the $B_{z}(x)$ peak due to the demagnetizing effects broadens and in the limit of $z \rightarrow \infty$, it should be recovered $B_{z}(x)=B_{\text {app. }}$. The asymmetric shape of the field profile at the border of the sample reflects the long range perturbation of the magnetic field outside the sample, caused by demagnetization effect at the border of the strip, which contrasts with a much sharper decay of the magnetic field upon entering the superconductor. More quantitatively, the magnetic field outside (inside) decreases halfway towards its final value $B_{z}=B_{\text {app }}\left(B_{z}=0\right)$ at a distance $\sim 7.5 \mu \mathrm{m}$ $(\sim 3 \mu \mathrm{m})$ from the border.

The fitting of the curves is based on Eq. (5) for $a=300 \mu \mathrm{m}$. All the curves are adjusted simultaneously with the respective scanning heights $z_{i}$ and a common value for $\Lambda$ as free parameters. The best fitting is obtained for $\Lambda=$ $715 \pm 300 \mathrm{~nm}$ and a lowest scanning height $z_{0}=1.38$ $\pm 0.15 \mu \mathrm{m}$. The resulting profiles are represented in Fig. 3(d) as continuous lines along with the corresponding values for $z_{i}=z_{0}+n p$, with $n$ integer. The modelled magnetic field profile evolution with $z$ is in good agreement with the experiment and the estimated value for the step of the vertical slider is around $0.9 \mu \mathrm{m}$, close to the expected value of $p=0.8 \mu \mathrm{m}$ provided by the manufacturer for $T=4.2 \mathrm{~K}$.

Knowing that $\Lambda=\lambda^{2} / t=715 \mathrm{~nm}$, we can estimate $\lambda(T=4.2 \mathrm{~K}) \approx 189 \mathrm{~nm}$, and assuming Ginzburg-Landau temperature dependence $\lambda(T)=\lambda(0) / \sqrt{1-T / T_{\mathrm{c}}}$, we deduce $\lambda(0) \approx 122 \mathrm{~nm}$. From the temperature dependence of the upper critical field, we can estimate a coherence length $\xi(0) \approx 33 \mathrm{~nm} \ll \xi_{0}=82 \mathrm{~nm}$ meaning that our $\mathrm{Pb}$ film falls in the dirty limit. Using the dirty limit correction for the penetration depth $\lambda=\left(0.64 \sqrt{\xi_{0} / \ell}\right)^{-1} \lambda_{\mathrm{L}}$ and coherence length $\xi(0)=0.855 \sqrt{\xi_{0} \ell}$, it is easy to show that $\lambda_{\mathrm{L}} \approx 1.83 \xi(0) \lambda(0) / \xi_{0} \sim 89 \mathrm{~nm}$. The fact that we have chosen to work at a field very close to the penetration field, $H_{\mathrm{p}}$, implies that the screening current is very close to the depairing current. ${ }^{14}$ It is worth noting that London and Ginzburg-Landau theories give similar results, except at the border of the sample when $H_{\text {app }}$ is close to $H \mathrm{p}$, which are the conditions of this experiment. ${ }^{18}$ Therefore, it is imperative to consider the kinetic suppression of the order parameter ignored within the London approximation but properly accounted in the Ginzburg-Landau formalism. This depletion of the superconducting condensate at the border of the sample leads to a less efficient screening manifested by a larger penetration depth $\lambda_{\text {edge }}=1.84 \lambda_{\mathrm{L}}$. This factor results from the decrease of the density of states with increasing current and is described within the first Ginzburg-Landau equation, which expresses conservation of energy and effectively couples the Cooper pair-density with the pair velocity. ${ }^{19}$ When taking into account this correction, we obtain $\lambda_{\mathrm{L}}=48 \pm 11 \mathrm{~nm}$, which is in reasonable agreement with
$\lambda_{\mathrm{L}}=40 \mathrm{~nm}$ obtained by alternative techniques. ${ }^{20}$ Notice that following a similar analysis and assuming that $\lambda_{\mathrm{L}}$ is known, the effective coherence length could be deduced from $\Lambda$.

\section{Effect of finite size of the sensor}

Equation (5) presumes an infinitely small magnetic sensor and therefore ignores the finite size $s$ of the active area of the Hall probe. Taking into account, this effects result in a convolution effect on the real magnetic field. In other words, instead of measuring $B_{z}(x, z)$, the measured field is given by

$$
\langle B\rangle=\int_{S} d A G(x, y) B(x, y),
$$

where $S$ is the active surface and $G$ is the response function accounting for the inhomogeneity of the sensitivity. Several references can be found that calculate $G$ depending on the kinetics of the electrons (diffusive regime, ${ }^{21,22}$ ballistic regime $^{23,24}$ ) and the size of the Hall probe. It is shown that when the Hall sensor operates in the ballistic regime and at low field, the response function can be considered as a constant within the main junction area, with rapid decaying tails in the contact paths and independent of the shape and position of the field inhomogeneity profile in the junction. Practically, the convolution product is computed numerically by averaging the field amplitude over the number of neighbouring pixels corresponding to $s$.

The effect of convolution by the size $s$ of the active area of the Hall probe is visible in Fig. 4. The value of $s=1 \mu \mathrm{m}$ is deliberately bigger than the real chip value (around $300 \mathrm{~nm}$ ) to emphasize the effects of convolution. These effects are important only where the field varies quickly as a function of $x$, i.e., near the peak. For a height $z=0.4 \mu \mathrm{m}$ significantly lower than the height at which the measurements were taken, the convolution decreases the amplitude of the peak by $6.1 \%$

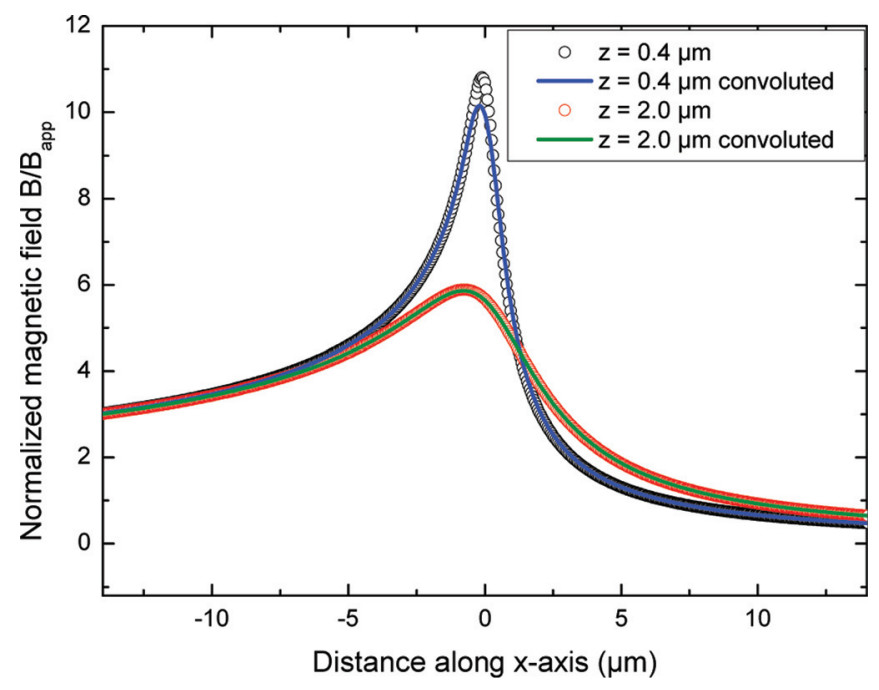

FIG. 4. Influence of the size $s$ of the active area of the Hall probe on the magnetic field profile. The fixed parameters are $\Lambda=1.2 \mu \mathrm{m}, a=300 \mu \mathrm{m}$, and $s=1 \mu \mathrm{m}$. The influence of convolution is only visible for the peak and at low $z$. For values of $z \gtrsim 1 \mu \mathrm{m}$ used in our experiment, convolution is negligible. 
compared to its initial value. For $z=2.0 \mu \mathrm{m}$, the influence of convolution is slender and the relative variation of the peak amplitude is of $0.4 \%$. By fixing $s=450 \mathrm{~nm}$, the convolution for $z=2 \mu \mathrm{m}$ is responsible for a relative amplitude variation of the peak smaller than $0.1 \%$. We can thus safely neglect the influence of the Hall probe size $s$ and the effect of convolution by the active area for our data.

\section{ISOLATED FLUX QUANTUM}

Let us now investigate the profile of an individual isolated vortex. To that end, we applied a magnetic field $H_{\text {app }} \ll H_{1}$ perpendicular to the sample surface, where $H_{1}=\Phi_{0} / w^{2}$ is the first matching field at which the density of vortices coincides with the density of antidots. In this case, after cooling down the sample to $4.2 \mathrm{~K}$ (field-cooling process), the vortices will be separated by a distance much larger than $\Lambda$. Thus, we can consider them as isolated, as shown in Fig. 5(a) for the minimal scanning height $z_{0}$. In order to extract the magnetic field profile from the isolated vortex, we consider the points located on the circumference of circles centred on the vortex and of radius $r$. We then
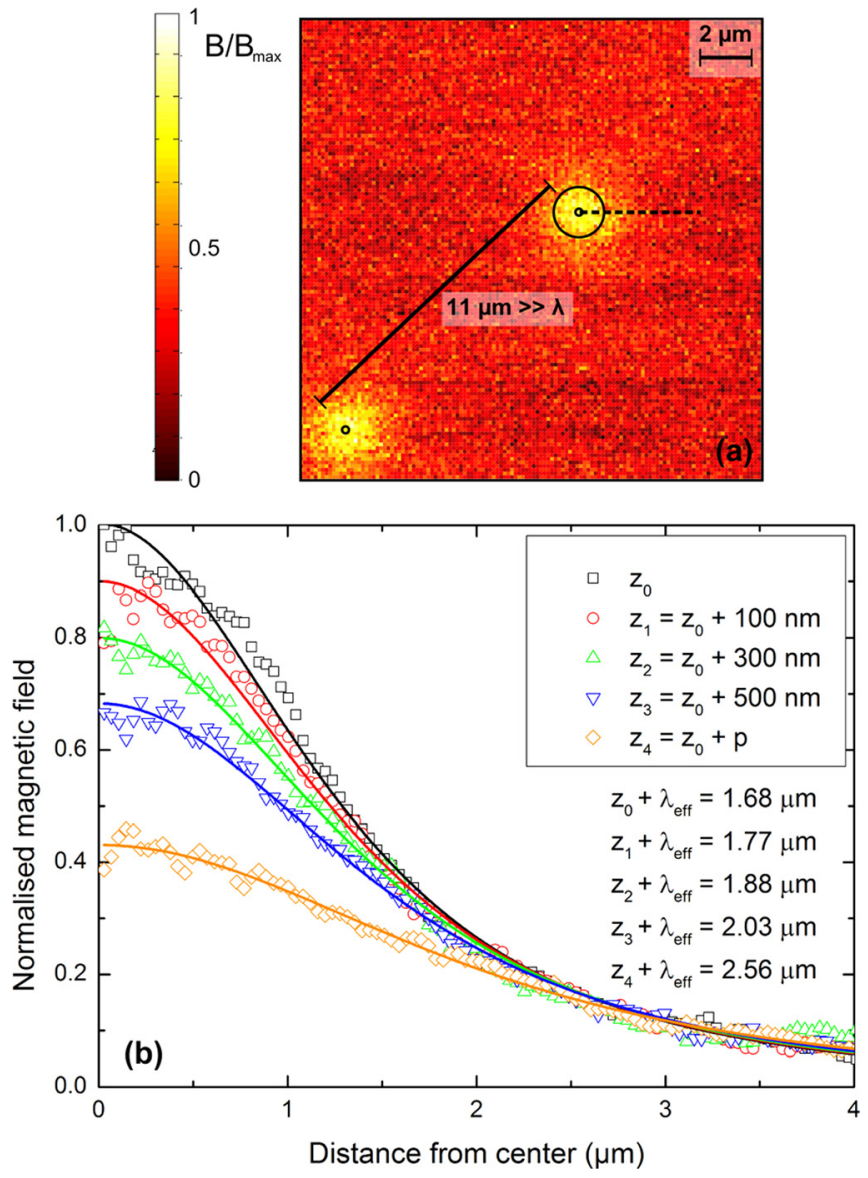

FIG. 5. (a) SHPM image at the middle of the superconducting strip after field cooling in a constant magnetic field. Two well separated vortices can be seen (bright spots). The values of the field were averaged on circles like the one shown in the figure. The dashed line shows the extension of the obtained field profile. (b) Evolution of the magnetic field profile of a single vortex as a function of the height $z$ of the probe. The plain curves represent the fitting based on the magnetic monopole model. This model yields values for $L=z+\lambda_{\text {eff }}$ for different $z$ that are consistent with the expected increase of $z$ between the curves. average the values of the field at these points for $r$ between 0 and $4 \mu \mathrm{m}$. We collected several SHPM images $B_{z}(x, y)$, at different scanheights $z$.

Theoretically, the field profile of a single vortex line can be described as the field produced by a magnetic monopole. ${ }^{25}$ This model is derived from the boundary condition at the superconducting-vacuum interface, where the London equations and the Maxwell equations must be satisfied, respectively, inside and outside the superconductor. It is valid for an infinite plane film with an arbitrary thickness. In the limit of $\sqrt{r^{2}+z^{2}} \gg \Lambda$, where $r$ is the in-plane distance measured from the center of the vortex and $z$ is the height measured from the surface, the magnetic field profile is described by the following expression:

$$
B_{z}(r, z)=\frac{\Phi_{0}}{2 \pi} \frac{z+\lambda_{\text {eff }}}{\left(r^{2}+\left(z+\lambda_{\text {eff }}\right)^{2}\right)^{3 / 2}},
$$

where $\lambda_{\text {eff }}=\lambda \operatorname{coth}(d / 2 \lambda)$. This equation represents the magnetic field of a magnetic monopole of amplitude $2 \Phi_{0}$ localized at a distance $\lambda_{\text {eff }}$ beneath the surface. We can consider the vortex as a monopole because the magnetic field is the same as if the flux lines where produced at one single point, and they do not close on themselves. ${ }^{26} \mathrm{We}$ use Eq. (7), where $\Phi_{0} / 2 \pi$ is replaced by a multiplicative constant, to represent the data. The fitting is done for all curves simultaneously with respect to this common constant and the values of $z_{i}+\lambda_{\text {eff }}$ for each curve, which are listed in Fig. 5(b). We can estimate the step $p$ of the vertical slider by comparing the values for the first and the last curves. This yields $p=0.88 \mu \mathrm{m}$. It confirms the value of $p=0.9 \mu \mathrm{m}$ found in the Meissner state and agrees with the theoretical value of $0.8 \mu \mathrm{m}$ given by the SHPM manufacturer.

From the fitting, we conclude that the magnetic monopole model correctly describes the magnetic field profile of an isolated vortex in a sample with a periodic array of holes. Unfortunately, we do not have access to the probe height $z$ and to $\lambda_{\text {eff }}$ separately, because these two parameters appear only through their sum in the theoretical relation. Thus, it is impossible to distinguish between a change in $z$ or $\lambda_{\text {eff }}$, as the effect on the magnetic field is the same. However, assuming $\lambda_{\text {eff }}=$ $\Lambda=715 \mathrm{~nm}$ as found in the Meissner state, ${ }^{27-29}$ we can extract the effective scanning heights $z_{i}$ and we find namely $z_{0}=0.98 \pm 0.3 \mu \mathrm{m}$. This value is smaller than $z_{0}=1.38 \mu \mathrm{m}$ found at the border of the sample in the Meissner state.

As we already anticipated in Sec. II, the value of $z_{0}$ depends on the location where the scanning takes place, since the presence of defects and asperities at the surface of the sample can give different offsets. Actually, atomic force microscopy images in our sample reveal the presence of about $300 \mathrm{~nm}$ thick resist leftovers at the border of the sample which are absent in the centre. This resist seems to be source of the discrepancy in our measurements of $z_{0}$ for these two locations.

\section{CONCLUSION}

In this study, we used SHPM to image the magnetic field landscape at the border of a $\mathrm{Pb}$ thin film. Measurements of 
the magnetic field in the Meissner state follow the spatial dependency proposed by Plourde et al. ${ }^{17}$ based on LondonMaxwell equations. A procedure is proposed which uses the theoretical fitting of these curves to determine the magnetic penetration depth and the probe-sample distance. By taking into account the temperature dependence of the penetration depth and the kinetic depletion of the order parameter, we obtained $\lambda_{\mathrm{L}}=48 \pm 11 \mathrm{~nm}$, which agrees well with values reported in literature for $\mathrm{Pb}$. We deduced that the separation between the Hall sensor and the sample surface is normally slightly larger than $1 \mu \mathrm{m}$ and we demonstrated that for these heights, the size of the Hall probe does not introduce significant corrections. In addition, we showed that the magnetic monopole model is appropriate to describe the field profile of a single vortex and we used the value of $\Lambda$ found in the Meissner state to extract the effective scanning height, a crucial parameter needed for obtaining quantitative values of the magnetic field.

\section{ACKNOWLEDGMENTS}

This work was partially supported by the Fonds de la Recherche Scientique-FNRS, the Methusalem Funding of the Flemish Government, the Fund for Scientic ResearchFlanders (FWO-Vlaanderen), and the ARC Grant 13/18-08 for Concerted Research Actions, financed by the WalloniaBrussels Federation. J.B. acknowledges support from FRS-FNRS (Research Fellowship). J.V.d.V. acknowledges support from FWO-Vl. The work of A.V.S. was partially supported by "Crédit de demurrage," U.Lg.

${ }^{1}$ R. Prozorov, R. W. Giannetta, A. Carrington, P. Fournier, R. L. Greene, P. Guptasarma, D. G. Hinks, and A. R. Banks, Appl. Phys. Lett. 77, 4202 (2000).

${ }^{2}$ M. Abdel-Hafiez, J. Ge, A. N. Vasiliev, D. A. Chareev, J. Van de Vondel, V. V. Moshchalkov, and A. V. Silhanek, Phys. Rev. B 88, 174512 (2013).

${ }^{3}$ A. T. Fiory, A. F. Hebard, P. M. Mankiewich, and R. E. Howard, Appl. Phys. Lett. 52, 2165 (1988)

${ }^{4}$ J. Mao, D. H. Wu, J. L. Peng, R. L. Greene, and S. M. Anlage, Phys. Rev. B $\mathbf{5 1 ,} 3316(1995)$
${ }^{5}$ D. N. Basov, R. Liang, D. A. Bonn, W. N. Hardy, B. Dabrowski, M. Quijada, D. B. Tanner, J. P. Rice, D. M. Ginsberg, and T. Timusk, Phys. Rev. Lett. 74, 598 (1995).

${ }^{6}$ J. E. Sonier, R. F. Kiefl, J. H. Brewer, D. A. Bonn, J. F. Carolan, K. H. Chow, P. Dosanjh, W. N. Hardy, Ruixing Liang, W. A. MacFarlane, P. Mendels, G. D. Morris, T. M. Riseman, and J. W. Schneider, Phys. Rev. Lett. 72, 744 (1994).

${ }^{7}$ L. Luan, O. M. Auslaender, T. M. Lippman, C. W. Hicks, B. Kalisky, J.-H. Chu, J. G. Analytis, I. R. Fisher, J. R. Kirtley, and K. A. Moler, Phys. Rev. B 81, 100501 (2010).

${ }^{8}$ L. Luan, T. M. Lippman, C. W. Hicks, J. A. Bert, O. M. Auslaender, J.-H. Chu, J. G. Analytis, I. R. Fisher, and K. A. Moler, Phys. Rev. Lett. 106, 067001 (2011).

${ }^{9}$ J. Kim, N. Haberkorn, S.-Z. Lin, L. Civale, E. Nazaretski, B. H. Moeckly, C. S. Yung, J. D. Thompson, and R. Movshovich, Phys. Rev. B 86, 24501 (2012).

${ }^{10}$ J. Kim, L. Civale, E. Nazaretski, N. Haberkorn, F. Ronning, A. S. Sefat, T. Tajima, B. H. Moeckly, J. D. Thompson, and R. Movshovich, Supercond. Sci. Technol. 25, 112001 (2012).

${ }^{11}$ A. Kohen, T. Proslier, T. Cren, Y. Noat, W. Sacks, H. Berger, and D. Roditchev, Phys. Rev. Lett. 97, 27001 (2006).

${ }^{12}$ J. R. Kirtley, C. C. Tsuei, F. Tafuri, P. G. Medaglia, P. Orgiani, and G. Balestrino, Supercond. Sci. Technol. 17, 217 (2004).

${ }^{13}$ J. Pearl, Appl. Phys. Lett. 5, 65 (1964).

${ }^{14}$ J. Gutierrez, B. Raes, J. Van de Vondel, A. V. Silhanek, R. B. G. Kramer, G. W. Ataklti, and V. V. Moshchalkov, Phys. Rev. B 88, 184504 (2013).

${ }^{15}$ S. J. Bending, Adv. Phys. 48, 449 (1999).

${ }^{16}$ B. Raes, Ph.D. thesis, Catholic University of Leuven (2013), pp. 186.

${ }^{17}$ B. L. T. Plourde, D. J. Van Harlingen, D. Y. Vodolazov, R. Besseling, M. B. S. Hesselberth, and P. H. Kes, Phys. Rev. B 64, 14503 (2001).

${ }^{18}$ D. Yu. Vodolazov and I. L. Maksimov, Physica C 349, 125 (2001).

${ }^{19}$ K. Fossheim and A. Sudbø, Superconductivity: Physics and Applications (John Wiley \& Sons, 2005), p. 234.

${ }^{20}$ M. P. Nutley, A. T. Boothroyd, C. Staddon, D. M. Paul, and J. Penfold, Phys. Rev. B 49, 15789 (1994).

${ }^{21}$ S. Bending and A. Oral, J. Appl. Phys. 81, 3721 (1997).

${ }^{22}$ Y. G. Cornelissens and F. M. Peeters, J. Appl. Phys. 92, 2006 (2002).

${ }^{23}$ F. M. Peeters and X. Q. Li, Appl. Phys. Lett. 72, 572 (1998).

${ }^{24}$ A. K. Geim, S. J. Bending, I. V. Grigorieva, and M. G. Blamire, Phys. Rev. B 49, 5749 (1994).

${ }^{25}$ A. M. Chang et al., Appl. Phys. Lett. 61, 1974 (1992).

${ }^{26}$ G. Carneiro and E. H. Brandt, Phys. Rev. B 61, 6370 (2000).

${ }^{27}$ In this approximation, we are neglecting the fact that the vortex is trapped in a hole and therefore $\lambda$ should increase by half the size of the hole. This effect will lead to an even smaller $z_{0}$.

${ }^{28}$ A. I. Buzdin, Phys. Rev. B 47, 11416 (1993).

${ }^{29}$ A. Wahl, V. Hardy, J. Provost, Ch. Simon, and A. Buzdin, Physica C 250, 163 (1995). 